\title{
Development of an artificial nose integrating NEMS and biological olfactory receptors
}

\author{
${ }^{1}$ G. Gomila, ${ }^{1}$ A. Errachid, ${ }^{1}$ F. Bessueille, ${ }^{1}$ O. Ruiz, ${ }^{2}$ E. Pajot, ${ }^{2}$ J. Minic, ${ }^{2}$ T. Gorojankina, ${ }^{2}$ R. \\ Salesse,,${ }^{3}$ G. Villanueva, ${ }^{3} \mathrm{~J}$. Bausells, ${ }^{4} \mathrm{C}$. Pennetta, ${ }^{4}$ E. Alfinito, ${ }^{4} \mathrm{~F}$. della Sala, ${ }^{4} \mathrm{~V}$. Akimov, ${ }^{4} \mathrm{~L}$. \\ Reggiani, ${ }^{5}$ Y. Hou, ${ }^{5}$ N. Jaffrezic, ${ }^{6}$ G. Ferrari, ${ }^{6}$ L. Fumigalli, ${ }^{6}$ M. Sampietro, ${ }^{1}$ J. Samitier \\ I Lab Nanobioengineering CREBEC Electronic Department, Barcelona University; ${ }^{2}$ Institut National Recherche \\ Agronomique INRA (France); ${ }^{3}$ IMB-Centro Nacional de Microelectrónica; ${ }^{4}$ Instituto Nazionale per la fisica de al \\ Materia Leche (Italy); ${ }^{5}$ Ecole Centrale de Lyon (France); ${ }^{6}$ Politécnico de Milano
}

\section{Abstract}

Microsystems' including biomolecules is currently a research field in fast development and expansion. By coupling biomolecules to nonbiological electronic transducers, molecular bioelectronic systems could one day be used in a wide variety of applications, including biosensors, biofuel cells and maybe even biocomputers. A major thrust in this framework is to integrate and process the chemical information at molecular level inside of a microsystem.

In this sense, the animal olfactory system represents the gold standard of biosensors with its capability to identify and discriminate thousands of odorant compounds.

In order to mimic the performances of natural olfactory biosensors, the overall objective of european project "SPOT-NOSED" is the development of a nanobiosensor array based on the electrical properties of single olfactory receptors. The nanobiosensor array will integrate a set of nanotransducers, each of which will consist of two noble metal nanoelectrodes with an single olfactory receptor anchored in between

\section{Introduction}

In the recent years important advances in biotechnology and nanotechnology have taken place, opening a way to the development of single biomolecule-based nanobiosensors. These nanobiosensors will represent the ultimate limit in miniaturisation, specificity and sensitivity, and would constitute the closest bio-electronic mimic of the animal sensing systems.
In the present RTD project we explore the possibility to develop the first olfactory nanobiosensor array based on the electrical properties of single olfactory receptors.

The nanobiosensor array will integrate a set of nanotransducers, each of which will consist of two functionalised metal nanoelectrodes with an olfactory receptor monolayer anchored in between. In order to reach this objective we will follow a cross-disciplinary approach in nanolithography, microelectronics, biochemistry and biotechnology.

The overall objective of the project is to explore the possibility to develop a nanobiosensor array based on the electrical properties of single olfactory receptors. The nanobiosensor array will integrate a set of nanotransducers, each of which will consist of two metal nanoelectrodes with an olfactory receptor monolayer anchored in between.

The main objective will be achieved through the following partial objectives: (i) the fabrication of low resolution nanoelectrodes, (ii) the expression and purification of a sufficient number and amount of olfactory receptors, (iii) the elaboration and transfer of monolayers of olfactory receptors on the metal nanoelectrodes, (iv) the characterisation and modelling of the electric properties of single olfactory receptors, (v) the determination of olfactory receptor-odour couples, and (vi) the design of a very low noise amplifying readout interface. 
The project will develop research in essentially four lines: (i) the production of the olfactory receptors and the elaboration of stable monolayers containing them, (ii) the fabrication of metal nanoelectrodes and the anchoring of olfactory receptors monolayers in between (nanotransducer) (iii) the characterisation of the electrical properties of single olfactory receptors and (iv) the characterisation of the nanotransducers as elementary nanobiosensors and their integration in an array biosensor.

We will start with olfactory receptors for which at least one ligand is know. At a later stage we will consider extracting olfactory receptors from a rat olfactory epithelium expression library so as to determine their odorant ligands, or even engineering the sequences of olfactory receptors.

The stable monolayers containing olfactory receptors will be formed either from purified olfactory receptors solubilised in a detergent or by incorporating them into a lipid membrane. The immobilisation will be enhanced with specific antibodies covalently bound on the nanoelectrodes.

The fabrication of the nanoelectrodes will be carried out by means of NanoImprint Lithography, assisted by Electron Beam Lithography and Reactive Ione Etching.

The electrical characterisation of single olfactory receptors will be performed by means of both a specifically designated atomic force microscope and by means of the nanotransducers, and will be assisted by the development of appropriate theoretical models.

The feasibility of the nanotransducers as nanobiosensors will be based on the changes in the electric properties of single olfactory receptors when an odour molecule is bound. vacuoles.
The corresponding detection curves for given couples of olfactory receptor-odour will be established. Finally, by implementing a set of nanobiosensors in a nanobiosensor array specific product detection will be essayed. Appropriate very low noise amplifiers will be used.

\section{Main results}

Different techniques of obtaining and purifying proteins have been tested. Olfactory receptor expression in yeast's plasmic membrane, obtains functional receptors.

This new technique is able to produce relatively large amounts of proteins. In our case, it has been tuned up for rat (17) and human (OR 17-40) olfactory receptors mass production and its high yielding has been observed by ELISA-type test. High-resolution electron microscopy shows that the receptors are located, basically, at the cell membrane. The presence of the 17 olfactory receptor was evident at the plasmic membrane showing that some synthesized 17 molecules are addressed to their functional localization.

The electrical characterization of single protein olfactory receptors is performed using nanotools. The nanotools are essentially atomic force microscopy (AFM) probes with the capability of conducting electrical current but able to be used in a liquid solution. This requires that an electrical insulator cover the probes except at the tip. This feature is not available in commercial AFM probes.

Figure 1: AFM Image of fraction membrane containing protein receptors. The size of objects about $40 \mathrm{~nm}$ correspond to small liposomes

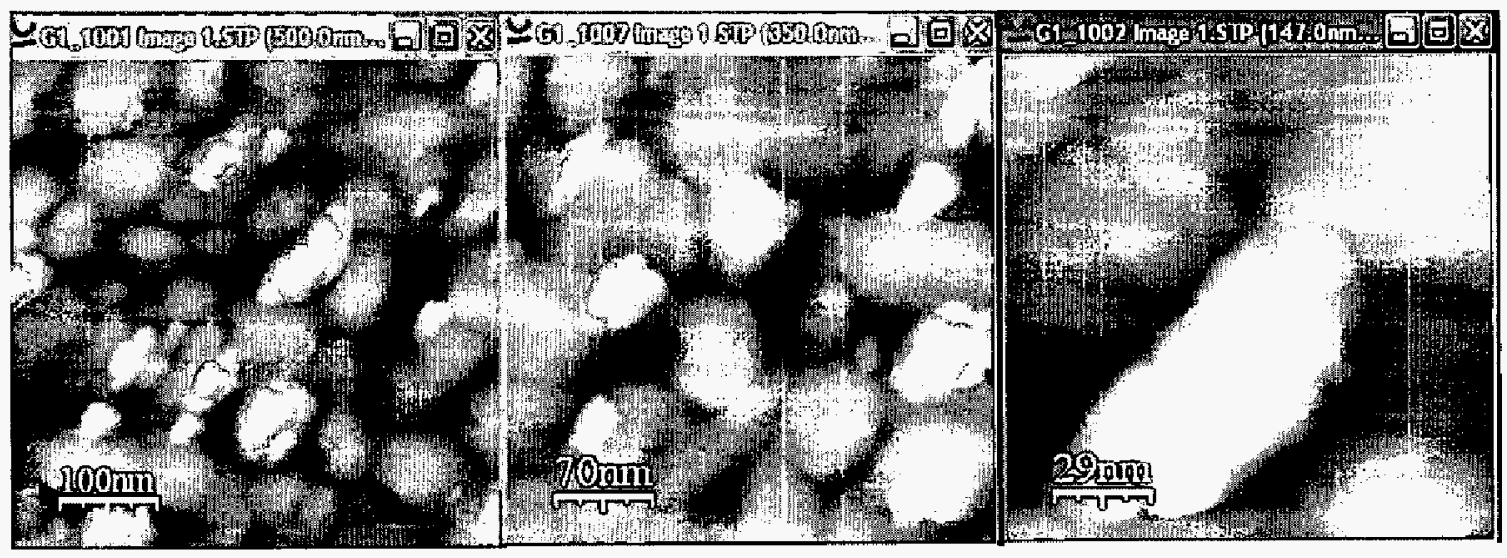


Relating to the transfer of proteins on substrates, a new technique based on mixed SAM and printing techniques (fig 2 and fig 3) was carried out in order to immobilize biotinylated specific mono or polyclonal antibody on gold surface and then to selectively fix olfactory receptor. This new technique improves the previous one in the cconomy of biological samples, the stability of the obtained structure and the control of the density of grafted biotinylated antibodies. The process include the use of microcontact printing ( $\mu \mathrm{CP}$ ) on reactive, mixed self-assembled monolayers (SAMs) of 16-mercaptohexadecanoic acid (MHDA) and Biotinyl-PE on gold to pattern ligands. The resulting SAMs present biotinylated sites, which can be used to construct self-assembly multilayers for biosensor research (fig 4). In our case, the multiplayer system consists of mixed SAMs, neutravidin, biotinylated antibody against I7 olfactory receptor, and membrane fragments including 17 olfactory receptors.

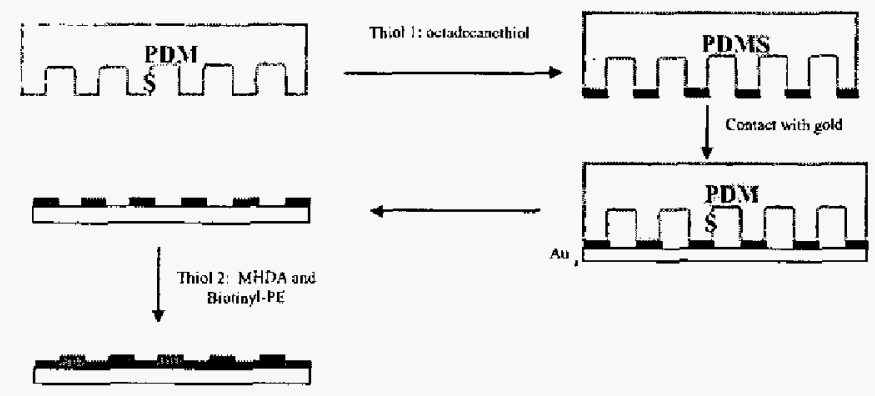

Figure 2. Double functionalization process using microcontact printing

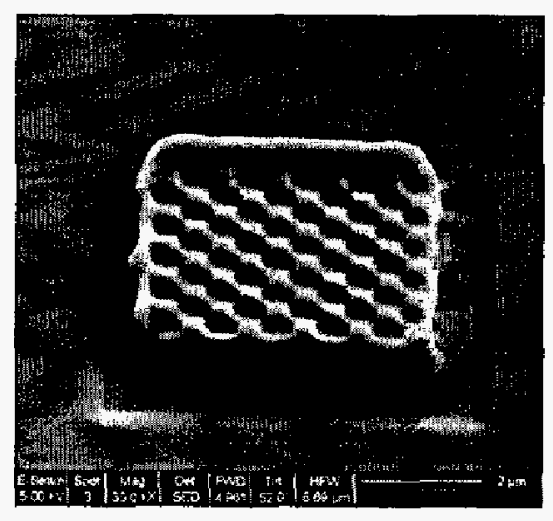

Figure 3: Master to perform the functionalization process obtained using FIB lithography.

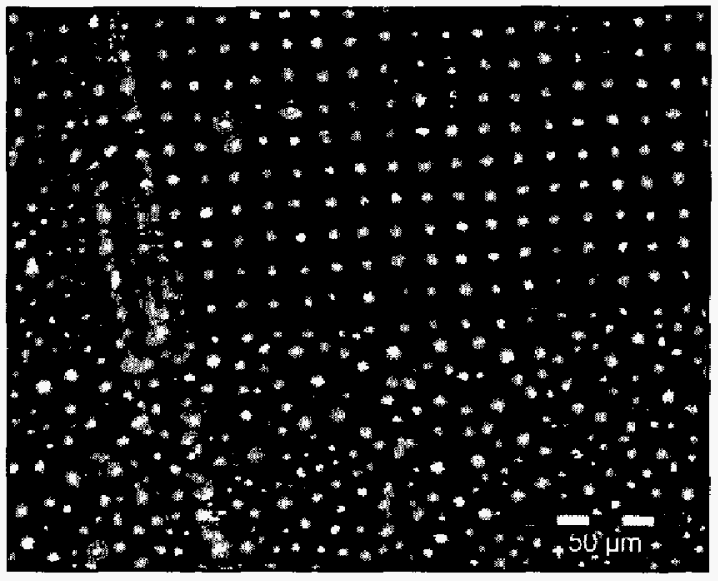

Figure 4 Example of functionalization process using printing. streptavidin Texas Red-X conjugate protein

The nanosystem array requires the nanolectrodes fabrication based on a combination of micro and nano-technologies, using e-beam and focused ion beam lithography (fig 5,6)

Electrical impedance measurements over ion sensitive microelectrodes evidence indicia that bring forth the interaction between the 17 olfactory receptor present in the deposited membrane fractions and the heptane as odorant. Measurements with the AFM of the electrical properties of a variety of molecular monolayers of interest for the development of the nanobiosensors (olfactory receptor monolayers, and functionalisation monolayers) have been also realized using four types of electrical measurement tests, namely, DC conductive maps, DC current-voltage characteristics, DC currentdistance characteristics and $A C$ small signal measurements. As showed in figure $7, \mathrm{DC}$ current-voltage characteristics present a tunnellike characteristics which can be reasonable well with the theoretical expression

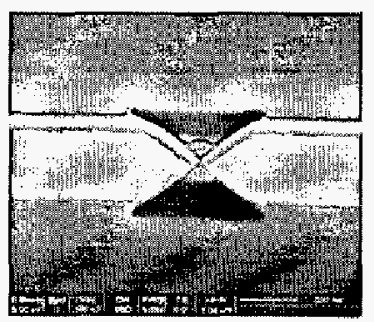

Figure 5 Photomicrograph of single nanoelectrodes ( $20 \mathrm{~nm}$ gap) obtained by FIB assisted deposition and etching 
(a)

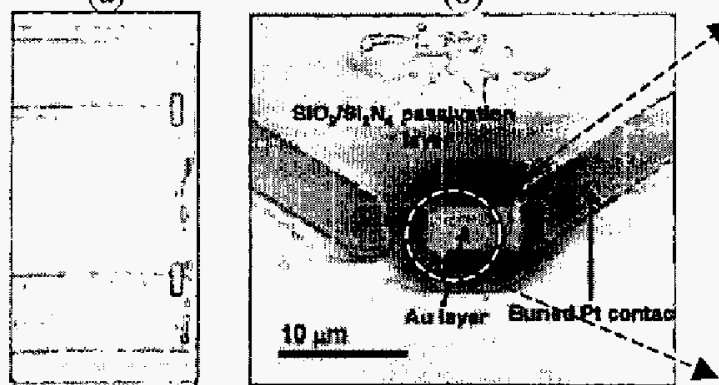

(c)

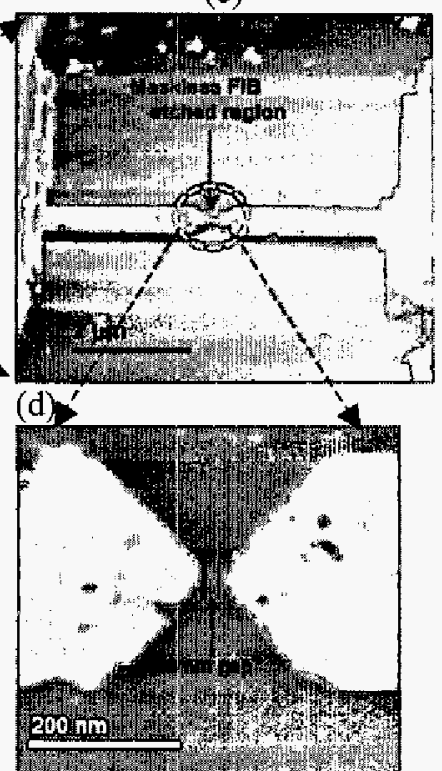

Figure 6 Picture corresponding to nanoelectrodes fabricated using micro and nanolithography. (b),(c),(d) SEM details showing som FIB lithography process: b) window in the passivation layer (c) metal layer etching, d) nanoelectrode gap $40 \mathrm{~nm}$.

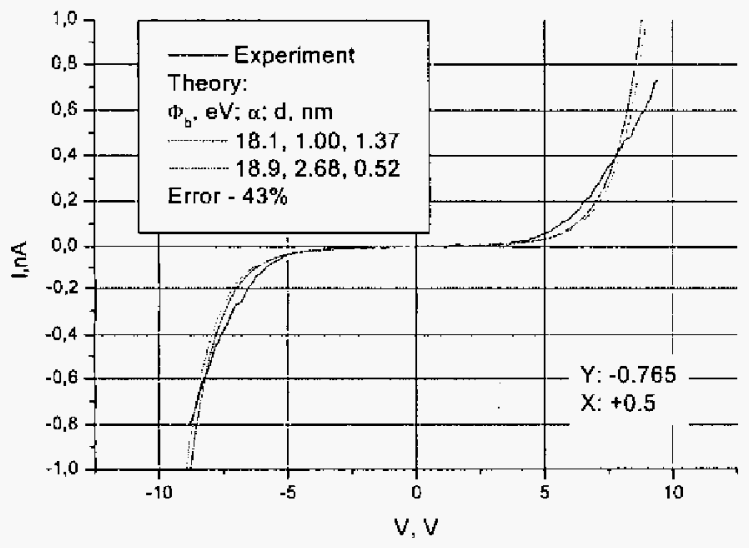

Figure 5 DC current-voltage characteristics obtained on point of a deposited multilayer with protein receptors on a gold substrate. The measurements fit well with the theoretical expression.

Acknowledgements: Spot-Nosed project IST2001-38899 and CICYT-TIC-2002-04280-C03 